\title{
Separation, Quantification, and Visualization of DNA
}

Zachary McLeod, Megan Bestwick

Department of Chemistry, Linfield College, McMinnville, Oregon 97128

\section{Introduction}

Mitochondria are involved in numerous essential cellular pathways. ${ }^{1}$ Mitochondria contain their own DNA genome (mtDNA), which is distinct from nuclear DNA. Scientists wish to understand the mitochondrial genome as a whole as well as how mitochondrial transcription is affected through changes in the proteins involved in regulation. The direct output of mitochondrial transcription is mitochondrial RNA (mtRNA), so separation and quantification of this mtRNA would be highly beneficial to this understanding. Ionpair reversed-phase high performance liquid chromatography (IP RP HPLC) has been reported to be an effective way to analyze DNA and RNA oligonucleotides (Model 1)., ${ }^{2,3}$ IP RP HPLC can analyze oligonucleotide samples in under an hour as opposed to agarose gel electrophoresis followed by ethidium bromide detection or P-32 labelling which both take up to 4-5 hours to fully analyze samples. 4,5 We report a novel IP RP HPLC method which separated ssDNA samples of $54 \mathrm{nt}$ and $58 \mathrm{nt}$ with good resolution. This method also quantified ssDNA samples of $54 \mathrm{nt}$ and $58 \mathrm{nt}$ upwards of $5000 \mathrm{ng}$. This method will be further optimized to separate similarly sized RNA samples. The ultimate goal is to separate mixtures of nucleotides generated from in-vitro transcription reactions. We also report a method for imaging DNA molecules immobilized on mica in air using atomic force microscopy (AFM).

\section{Methods and Materials}

Diethyl pyrocarbonate (DEPC)-treated water was used for all oligonucleotide sample preparation. Samples were obtained lyophilized, and dissolved in sufficient DEPC-treated water such that the final concentration was $100 \mathrm{ng} / \mu \mathrm{L}$.

Ion-pair reversed-phase high performance liquid chromatography (IP RP HPLC) was performed on a Shimadzu LC$2010 \mathrm{HT}$ Liquid Chromatography System with an Xbridge Oligonucleotide BEH C18 (130 A, $2.5 \mu \mathrm{m}, 4.6$ x $50 \mathrm{~mm}$ ) column. Quantification of peaks was performed through integration of peak areas.

- Buffer A: 0.1 M TEAA in DEPC-treated water (pH 7.0)

- Buffer B: 0.1 M TEAA with 25\% (v/v) ACN in DEPC-treated water ( $\mathrm{pH}$ 7.0)

The gradient conditions used were: $38-39 \% \mathrm{~B}$ from 0 to 45 minutes, to $40 \%$ B from 45 to 55 minutes, to $100 \%$ B from 55 to 60 minutes, followed by a 10-minute re-equilibration to initial conditions. Flow rate was $0.5 \mathrm{~mL} / \mathrm{min}$. Column temperature was kept at $50^{\circ} \mathrm{C}$. Oligonucleotide samples were kept at $4{ }^{\circ} \mathrm{C}$ prior to analysis to prevent sample degradation.

Mica discs were epoxied to magnetic pucks and freshly cleaved with tape creating a near atomically flat surface. $50 \mu \mathrm{L}$ of DNA was pipetted onto freshly cleaved mica discs and dried under nitrogen.

AFM imaging of DNA oligos was performed on an Asylum Research Cypher Scanning Probe Microscope. The microscope was operated in air tapping mode using cantilever oscillation frequencies between 850 and $2500 \mathrm{kHz}$. The lever used was a chromium/gold coated silicon lever with a $7 \mathrm{~nm}$ silicon tip and a spring constant of $85 \mathrm{~N} / \mathrm{m}$

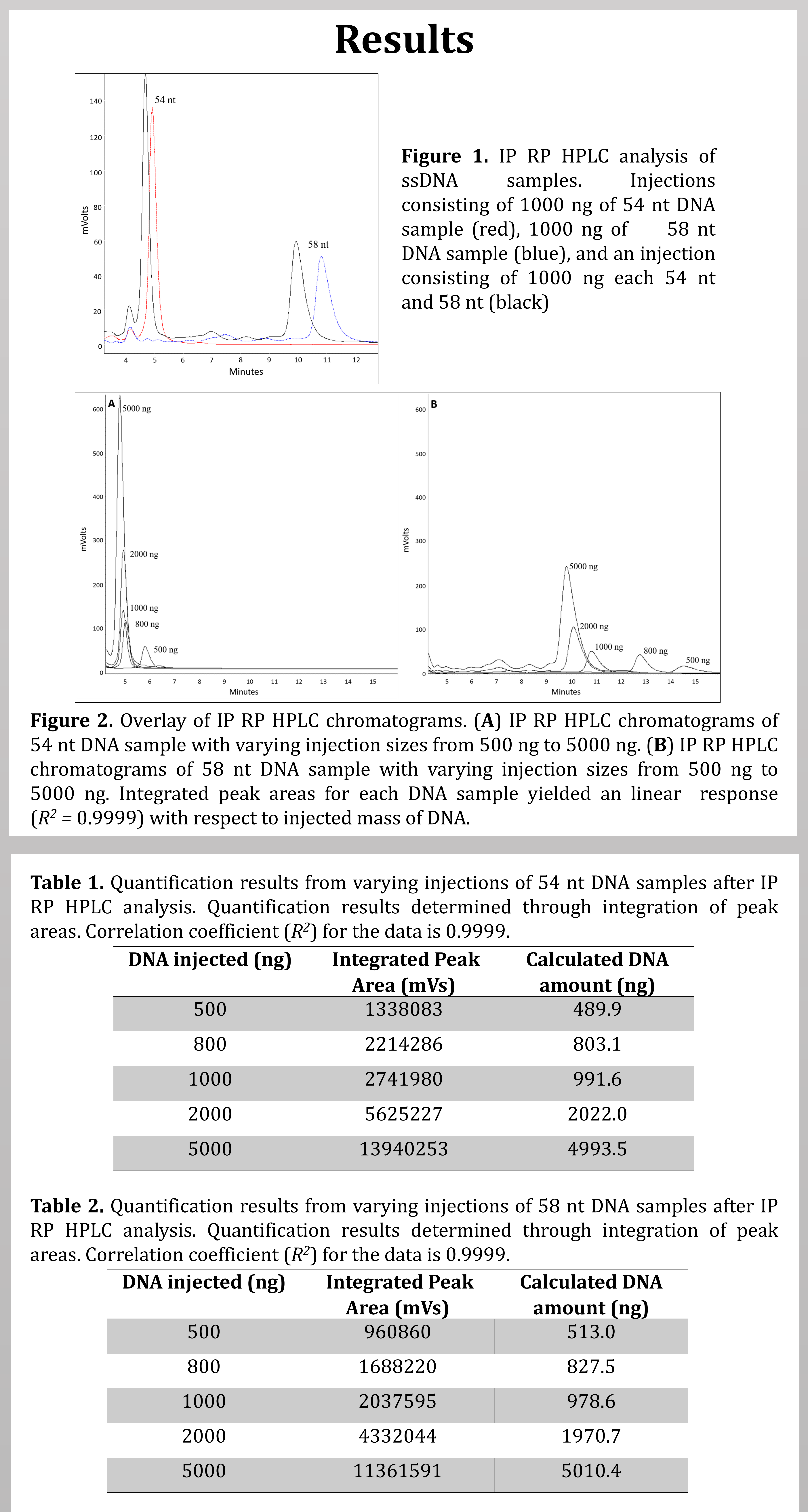

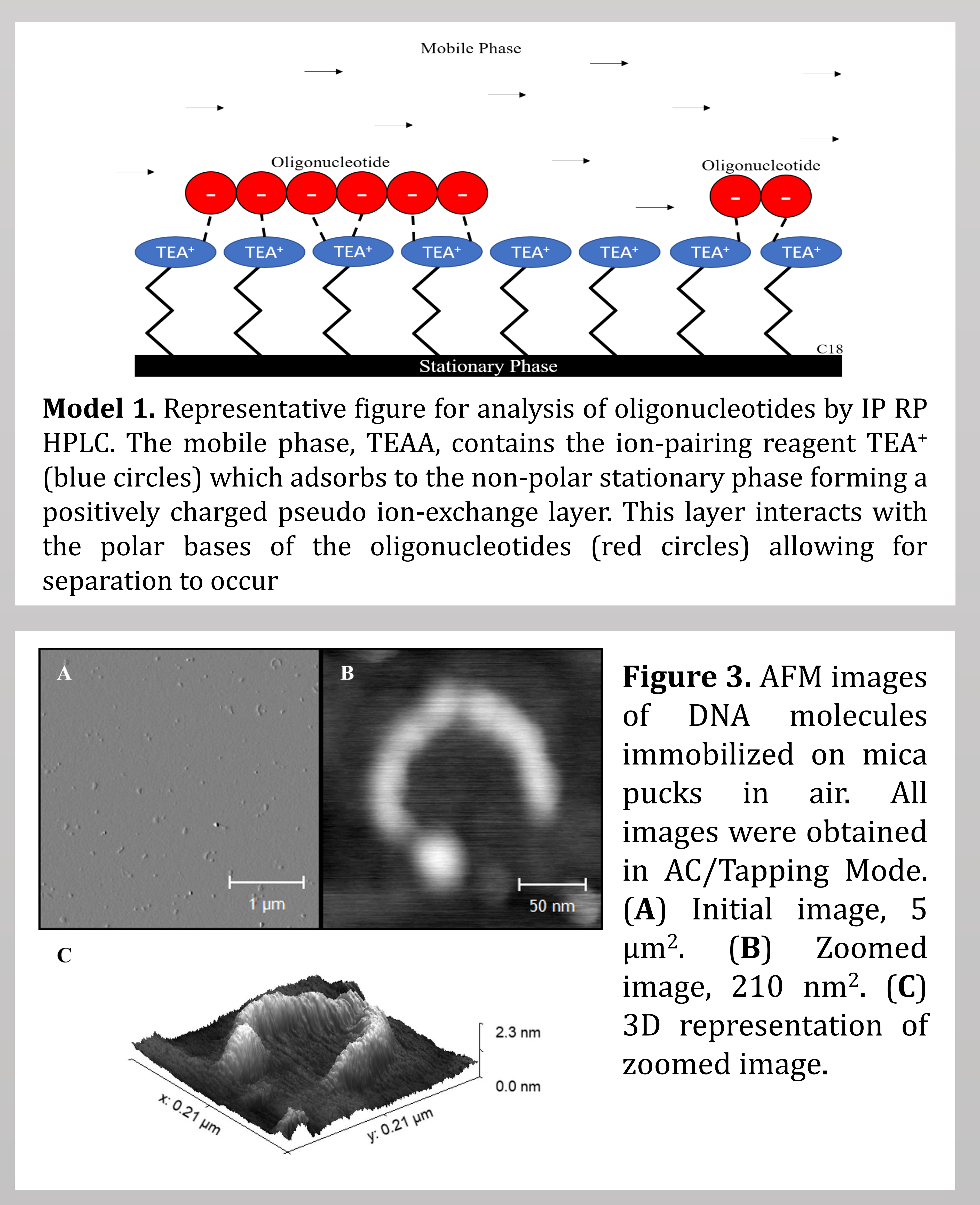

\section{Future Directions}

Going forward we hope to optimize the current method and apply it to separate RNA samples of similar sizes to the DNA samples tested. The ultimate goal is to utilize this method and IP RP HPLC to separate and quantify RNA transcripts of unknown sizes generated via in-vitro transcription reactions. We also want to improve AFM image quality, and shift the focus to imaging DNA samples and DNA-protein samples in liquid. From there, our aim is to determine force constants of protein-DNA interactions using functionalized lever tips.

\section{Acknowledgements}

Linfield Chemistry Department, Linfield College Student Faculty Collaborative Research," National Science Foundation (MRI-DMR \#1827971), Murdock Charitable Trust (Natural S \#2015313), Linfield Physics Department, Dr. Jennifer Heath, Joseph Simpson, Wendell L. Foote Fund

\section{References}

${ }^{1}$ Bestwick, M.L., et al. (2013) Trends Biochem Sci, 38, 283-291 ${ }^{2}$ Wysoczynski, C.L., et al. (2013) Nuc Acids Res, 41 e194

${ }^{3}$ Azarani, A., et al. (2001) Nuc Acids Res, 29, e7

${ }^{4}$ Lee P.Y., et al. (2012) J Vis Exp, 62, e3923

${ }^{5}$ Shutt, T.E., et al. (2010) Proc Natl Acad Sci USA, 107 (27), 12133-12138 